\title{
Two Kondo Impurities in Armchair Graphene Nanoribbon
}

\author{
S. LIPIŃSKI AND D. KRYCHOWSKI \\ Institute of Molecular Physics, Polish Academy of Sciences, M. Smoluchowskiego 17, 60-179 Poznań, Poland
}

An interplay of the Kondo effect and inter-impurity correlations in the armchair graphene nanoribbon is studied in the mean field slave boson approach. The effect of the hole doping is examined.

PACS: 73.22.Pr, 75.20.Hr, 75.30.Et

\section{Introduction}

A band gap in graphene can be created by its patterning into nanoribbon. The electronic structure of nanometer-wide nanoribbon with well defined edge symmetries are dominated by confinement effects and Van Hove singularities. As an example we will discuss the infinite long armchair ribbon composed of seven carbon dimer lines (7-AGNR, see the inset of Fig. 1). Due to the confinement, the spectrum breaks into a set of subbands $(2 N=14)$ and the wave vector along the confined direction becomes discretized. The aim of the present paper is to analyze the interplay between exchange interaction and the Kondo effect of a pair of magnetic impurities adsorbed on top of AGNR carbon atoms. Magnetic moment of the impurity, contact interaction between impurity spin and nanoribbon electrons as well as effective exchange interaction between the moments depends on geometrical position of impurities and gate voltage. The carrier number of AGNR can be manipulated with gate voltage. We discuss the case of hole doping.

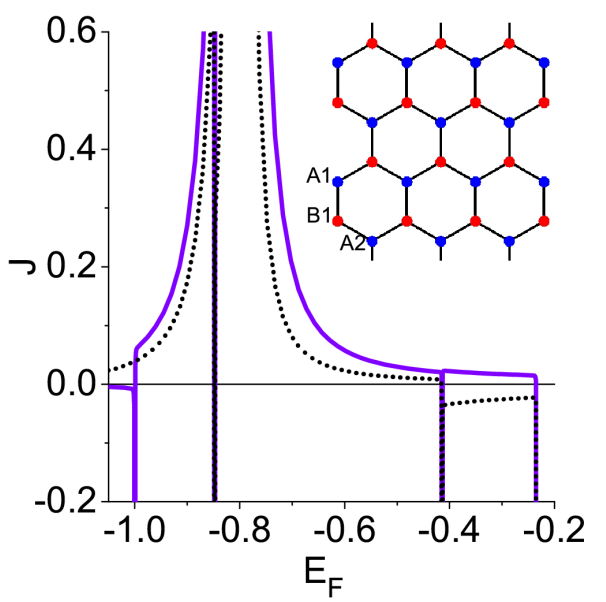

Fig. 1. Nearest neighbor $\left(\mathcal{J}_{\mathrm{A} 1-\mathrm{B} 1}-\right.$ solid line $)$ and next nearest neighbor $\left(\mathcal{J}_{\mathrm{A} 1-\mathrm{A} 2}-\right.$ dotted line) exchange integrals of hole doped 7-AGNR versus position of the Fermi level. Inset shows considered armchair graphene nanoribbon with the marked sites of interest.

\section{Model}

We describe the electronic states of AGNR by the tight-binding model (TB):

$$
\mathcal{H}_{\mathrm{GNR}}=-t \sum_{i j \sigma}\left(c_{i \sigma}^{\dagger} c_{j \sigma}+\text { H.c. }\right) \text {, }
$$

where $c_{i \sigma}^{\dagger}$ denotes creation operator of $\pi$-electron on the site $i=\left(i_{x}, \alpha, i_{y}\right)$, where $x$ is confinement direction $i_{x}=$ $1,2, \ldots N$, and $\alpha$ labels graphene sublattices $\alpha=\mathrm{A}, \mathrm{B}$. The TB model with constant nearest-neighbor integral $t=2.7 \mathrm{eV}$ reasonably well reproduces AGNRs graphene band structure calculated in the local spin density approximation (LSD), with the exception for $N=3 p+2$, where in contrast to LSD predictions no band gap results [1]. A reasonable and simplest way within TB scheme to overcome this discrepancy is to introduce distortion induced changes of hopping integrals at the edges and then gap appears for any $N$ [1]. The case considered however $(N=7)$ does not fall into this category and therefore we omit this straightforward generalization, as giving only minor quantitative changes of the results. The impurities are considered in the Anderson approach

$$
\begin{aligned}
\mathcal{H} & =\sum_{\sigma} E_{0} d_{1 \sigma}^{\dagger} d_{1 \sigma}+\mathcal{U} n_{1+} n_{1-} \\
& +\sum_{\sigma} V\left(c_{i_{1} \sigma}^{\dagger} d_{1 \sigma}+\text { H.c. }\right)+\mathcal{H}_{\mathrm{GNR}},
\end{aligned}
$$

where the first two terms describe interacting localized electrons at impurity and the third hybridization with nanoribbon electrons, $i_{1}$ denotes the AGNR site that is closest to the adsorbed impurity. For $3 d$ elements in graphene the estimated values of $\mathcal{U}$ are around $2-4 \mathrm{eV}$ $[2,3], V$ is of order of $1-1.5 \mathrm{eV}[2,4]$ and impurity on-site energies $E_{0}$ ranges in the interval $(-3,-0.5) \mathrm{eV}$ $[2-4]$. Hereafter $t$ is used as the energy unit and we choose $E_{0}=-0.8, U=-2 E_{0}=1.6$ and $V=0.5$. The necessary requirement for the occurrence of the Kondo effect and magnetic coupling is that the impurities retain their magnetic moments in the presence of AGNR electrons. We do not consider here the subtle problem of moment formation, it is known that it is easier to generate magnetic moment in graphene than in an ordinary metal [5], we have only checked that in the Hartree-Fock approxima- 
tion the magnetic moments of impurities do not vanish in the considered parameter range. The exchange interaction $\mathcal{H}_{\alpha \beta}\left(R_{1}, R_{2}\right)=\mathcal{J}_{\alpha \beta}\left(R_{1}, R_{2}\right) S_{1} S_{2}$ may be written

$$
\begin{aligned}
& \mathcal{J}_{\alpha \beta}=-\frac{\left(\hbar J_{0}\right)^{2}}{2 \pi} \int_{-\infty}^{E_{\mathrm{F}}} \mathrm{d} \omega \operatorname{Im}\left(G_{\alpha \beta}^{0}\left(\omega, R_{1}, R_{2}\right)\right. \\
& \left.\quad \times G_{\beta \alpha}^{0}\left(\omega, R_{2}, R_{1}\right)\right),
\end{aligned}
$$

where $G^{0}$ are unperturbed retarded Green's functions of AGNR and contact interaction $\mathcal{J}_{0}=\left(V^{2} \mathcal{U}\right) /\left[\left(E_{0}-\right.\right.$ $\left.\left.E_{\mathrm{F}}\right)\left(E_{0}+\mathcal{U}-E_{\mathrm{F}}\right)\right]$. We set $\hbar=k_{\mathrm{B}}=1$.

\section{Results}

Figure 1 presents exchange integrals for impurity positions shown in the inset. We discuss the case, when chemical potential is located in the range of two highest conduction bands. The observed sharp jumps of exchange integrals occur close to the Van Hove (VH) singularities and for the Fermi level lying at the center of VHs, regardless on the positions of impurity sites, exchange integrals become ferromagnetic. A precise estimation of exchange integrals in this region certainly would require going beyond linear response theory, but the conclusion on the sign of integrals seems to be supported by similar observations for nanoscopic systems for the case of the Fermi level lying on one of resonant states of the host [6]. Despite the broken electron-hole symmetry for $E_{\mathrm{F}} \neq 0$, nearest neighbor exchange integral $\mathcal{J}_{\mathrm{A} 1-\mathrm{B} 1}$, similarly to the undoped graphene case, remains antiferromagnetic, except singularity points. Also similarly to undoped graphene inter-lattice integral $\mathcal{J}_{\mathrm{A} 1-\mathrm{A} 2}$ is ferromagnetic in the wide range of $E_{\mathrm{F}}$, but for energies below second singularity VH2 $\left(E_{\mathrm{F}}<-0.4142\right)$ it changes sign. To discuss the Kondo effect we have used slave boson (SB) approach in its simplest $\mathcal{U} \rightarrow+\infty$ formulation [7]. First let us consider the single impurity case. The slave boson operator $b^{\dagger}$ creates the empty state at the impurity and pseudofermion operator $f_{1 \sigma}^{\dagger}$ creates a singly occupied state. In the slave-boson mean field approximation (SBMFA) the boson field is replaced by its expectation value. The SBMFA Hamiltonian reads

$$
\begin{aligned}
\mathcal{H} & =\sum_{\sigma}\left(E_{0}+\lambda\right) f_{1 \sigma}^{\dagger} f_{1 \sigma}+\sum_{\sigma} V\left(b c_{i_{1} \sigma}^{\dagger} f_{1 \sigma}+\text { H.c. }\right) \\
& +\lambda\left(b^{2}-1\right)+\mathcal{H}_{\mathrm{GNR}} .
\end{aligned}
$$

The term with Lagrange multiplier $\lambda$ prevents double occupancy. The parameters $b$ and $\lambda$ are determined minimizing the ground state energy of (4).

Figure 2 displays evolution of the Kondo resonance with the shift of the Fermi level. In the region of smooth DOS of AGNR between VH singularities $\left(E_{\mathrm{F}}=\right.$ $\left.-0.28, E_{\mathrm{F}}=-0.3\right)$ the decrease of the Kondo temperature with lowering $E_{\mathrm{F}}$ can be understood solely as an effect of decreasing DOS of the host i.e. weaker coupling with impurity. Close to singularities the Kondo temperature enormously rises due to the strongly enhanced DOS of the host and the structure of the many-body

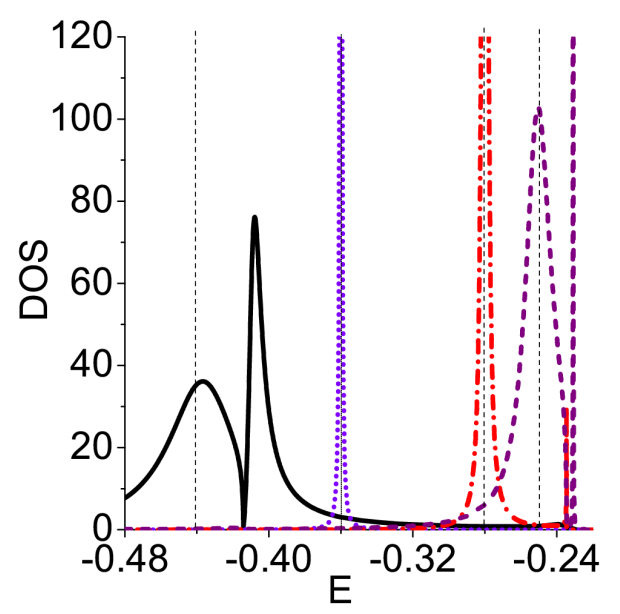

Fig. 2. Representative single impurity Kondo densities of states for adatom located on top of A1 or B1 sites for the chosen values of the Fermi energy (vertical dashed lines: $\left.E_{\mathrm{F}}=-0.44,-0.36,-0.28,-0.25\right)$.

resonance in this range is strongly influenced by resonant like hybridization (enhanced real part of AGNR self-energy). For the relatively deep position of impurity level, as it is the case for the Fermi level lying close to VH1 $\left(E_{\mathrm{F}}=-0.25\right)$, the two pole structure of the many-body resonance is observed with $\delta$-like peak in the gap. For the Fermi level placed in vicinity of lower singularity VH2 $\left(E_{\mathrm{F}}=-0.44\right)$ a dip in many-body resonance is observed. Atomic level in this case is closer to $E_{\mathrm{F}}$ and impurity is in mixed valence state. The SBMFA calculations in this range should be taken with caution $\left(b^{2} \approx 0.2\right)$ and they play only the qualitative role for visualization of tendency. To get the detailed information on the structure of the many-body resonances in this region and speculate e.g. on the deviations from the Fermi liquid picture certainly more rigorous calculations going beyond SBMFA are desired [8]. The estimated Kondo temperatures for A1 or B1 positions for the Fermi level placed between singularities ranges from $3 \mathrm{mK}$ to $1700 \mathrm{~K}$. In the regions of VH singularities the Kondo temperatures are strongly enhanced.

Before discussing magnetic interaction effect let us first show that even neglecting exchange coupling $\left(\mathcal{J}_{\mathrm{A} 1-\mathrm{B} 1}=\right.$ $0)$ the presence of another impurity can strongly reduce the Kondo temperature. Formally the extension of Hamiltonian (2) to the two impurity case requires introduction of additional slave boson operator for the second impurity $b_{2}$, supplement of (2) by hybridization term for second impurity and introduction of additional Lagrange condition with $\lambda_{2}$.

We present in Fig. 3 results only for symmetric case when impurities are located above n.n. carbon atoms (A1, B1) $\left(b_{1}=b_{2}, \lambda_{1}=\lambda_{2}\right)$. The Kondo peaks get narrow which corresponds to the decrease of $T_{\mathrm{K}}$ in comparison to single impurity case. Very roughly one can think that since the Kondo effect on impurity sitting on 


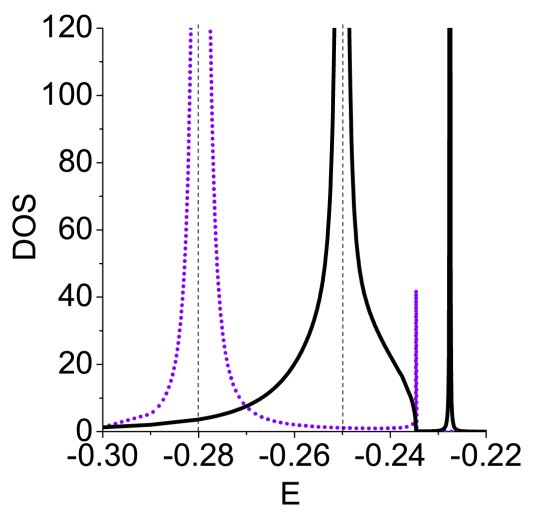

Fig. 3. Density of states of the Kondo dimers sitting on top of A1 and B1 sites for two values of the Fermi energy calculated for $\mathcal{J}_{\mathrm{A} 1-\mathrm{B} 1}=0$ (vertical dashed lines $\left.E_{\mathrm{F}}=-0.28,-0.25\right)$.

top of A1 induces a dip in DOS of A1 (the Kondo-Fano resonance), effectively it weakens coupling of B1 with AGNR conduction electron bath and consequently lowers $T_{\mathrm{K}}$. Similar arguing can be repeated choosing A1 instead of B1.

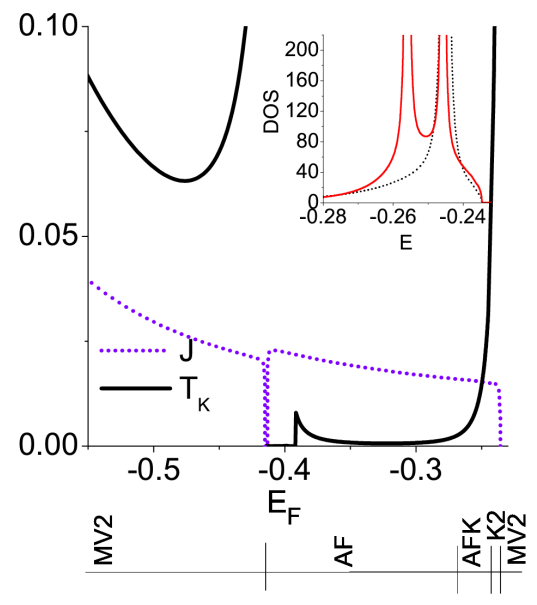

Fig. 4. Comparison of two-site Kondo temperature $T_{\mathrm{A} 1-\mathrm{B} 1}^{\mathrm{K}}\left(E_{\mathrm{F}}\right)\left(\mathcal{J}_{\mathrm{A} 1-\mathrm{B} 1}=0\right)$ and exchange integral $\mathcal{J}_{\mathrm{A} 1-\mathrm{B} 1}\left(E_{\mathrm{F}}\right)$. MV2, K2, AFK and AF denote respective intervals where according to mean field approximation mixed valence, pure Kondo, coexistence of the Kondo effect and magnetic ordering or pure magnetic ordering are expected. Inset shows representative DOS for K2 and AFK cases $\left(E_{\mathrm{F}}=-0.25,-0.245\right)$.

Figure 4 presents comparison of the Kondo temperatures with the exchange integrals. The detailed analysis of ordering tendencies is postponed for the future publi- cation [9]. Here we only show some preliminary results obtained treating exchange interaction within mean-field approximation: $\mathcal{J}_{\mathrm{A} 1-\mathrm{B} 1} S_{1} S_{2} \approx \mathcal{J}_{\mathrm{A} 1-\mathrm{B} 1}\left(S_{1}\left\langle S_{2}\right\rangle+\right.$ $\left.\left\langle S_{1}\right\rangle S_{2}-\left\langle S_{1}\right\rangle\left\langle S_{2}\right\rangle\right)$. The expectation values $\left\langle S_{1}^{z}\right\rangle=-\left\langle S_{2}^{z}\right\rangle$ are found self-consistently together with SBMFA parameters minimizing ground-state energy. Four regions can be distinguished: two-impurity Kondo state (K2) without magnetic ordering $\left(\left\langle S_{1}^{z}\right\rangle=0, b^{2} \neq 0, b^{2}<0.15\right)$, mixed valence state (MV2) $\left(\left\langle S_{1}^{z}\right\rangle=0, b^{2}>0.15\right)$, coexistence of the Kondo effect and AF correlations (AFK) $\left(\left\langle S_{1}^{z}\right\rangle=-\left\langle S_{2}^{z}\right\rangle, b^{2} \neq 0\right)$, and the range where the Kondo correlations are destroyed by exchange interactions (AF) $\left(b^{2}=0\right)$. The latter case manifests in SBMFA formalism by lack of solutions. The intervals of corresponding mean field solutions are marked on the picture. Inset shows representative DOS for the cases K2 and AFK.

\section{Conclusions}

In summary, present investigation only signals the richness of interplay of magnetic ordering tendencies and the Kondo correlations in graphene nanoribbons bringing out the role of confinement induced Van Hove singularities. The drastic changes around singularities suggest possibility to control magnetic behavior by applied gate voltage.

\section{Acknowledgments}

This work was supported by the Polish Ministry of Science and Higher Education as a research project No. N N202199239 in years 2010-2013.

\section{References}

[1] Y.W. Son, M. Cohen, S.G. Louie, Phys. Rev. Lett. 97, 216803 (2006).

[2] T.O. Wehling, A.V. Balatsky, M.I Katsnelson, A.I. Lichtenstein, A. Rosch, Phys. Rev. B 81, 115427 (2010).

[3] D. Jacob, G. Kotliar, Phys. Rev. B 82, 085423 (2010).

[4] P.S. Cornaglia, G. Usaj, C.A. Balseiro, Phys. Rev. Lett. 102, 046801 (2009).

[5] A.H. Castro Neto, V.N. Kotov, J. Nilsson, V.M. Pereira, N.M.R. Peres, B. Uchoa, Solid State Commun. 149, 1094 (2009).

[6] S. Galkin, C.A. Balseiro, M. Avignon, Eur. Phys. J. B 38, 519 (2004).

[7] P. Coleman, Phys. Rev. B 29, 3035 (1984).

[8] V.Yu. Irkhin, J. Phys., Condens. Matter 23, 065602 (2011).

[9] D. Krychowski, S. Lipiński, to be published. 